\title{
Comparing the Efficacy and Side Effects of PDLASTA® (Peg-Filgrastim) with PDGRASTIM® (Filgrastim) in Breast Cancer Patients; A Non-inferiority Randomized Clinical Trial
}

Safa Najafi

Breast Cancer Research Center, ACECR

Maryam Ansari

Breast Cancer Research Center, ACECR

Vahid Kaveh

Iran University of Medical Sciences: Tehran University of Medical Sciences

Shahpar Haghighat ( $\sim$ sha_haghighat@yahoo.com )

Breast Cancer Research Center,ACECR https://orcid.org/0000-0001-6938-467X

Research article

Keywords: Peg-Filgrastim, Filgrastim, chemotherapy, breast cancer, Iran

Posted Date: October 9th, 2020

DOI: https://doi.org/10.21203/rs.3.rs-78768/v1

License: (c) (i) This work is licensed under a Creative Commons Attribution 4.0 International License. Read Full License

Version of Record: A version of this preprint was published at BMC Cancer on April 23rd, 2021. See the published version at https://doi.org/10.1186/s12885-021-08197-6. 


\section{Abstract}

Background: The objective of this study was to compare the efficacy and side effects of to evaluate the efficacy and safety of a single dose (Peg-Filgrastim or PDL) or repeated six daily injections (Filgrastim or PDG) during chemotherapy courses in breast cancer patients in a non-inferiority clinical trial.

Methods: In this randomized clinical trial, 80 patients recruited and allocated randomly in two equal arms. In one group, a single subcutaneous dose of $6 \mathrm{mg}$ of PDL was injected the day after receiving a chemotherapy regimen in each cycle. The second arm received a subcutaneous injection of 300 micrograms per day for six consecutive days in each course of treatment. Side effects of GCF treatment and its effect on blood parameters were compared in each cycle and during eight courses of chemotherapy.

Results: hematologic parameters showed no significant difference in each course of treatment between two groups of study. The comparison of the WBC $(p=0.527), \mathrm{Hgb}(p=0.075)$, Platelet $(p=0.819)$, Neutrophil $(p=0.575)$, Lymphocyte $(p=705)$ and ANC $(p=0.675)$ changes during eight courses of treatment identified no statistically significant difference between two study groups. Side effects including headache, injection site reaction and muscle pain had a lower frequency in patients receiving PDL drugs.

Conclusion: Regarding our results, PDL is completely non- inferior in efficacy and also less toxic compared to PDG. Prescribing in single-dose and lower expenses of PDL introduces it as a cost-effective drug in the treatment of chemotherapy-induced neutropenia.

Trial registration number, date of registration: IRCT20190504043465N1, May 2019

https://www.irct.ir/search/result?query=IRCT20190504043465N1

\section{Background}

G-CSF is the main cytokine in the control of neutrophil production, which is used clinically for the treatment of congenital and acquired neutropenia[1]. This cytokine in vitro increases the number of circulating neutrophils and improves their performance[2]. More than $90 \%$ of patients respond to G-CSF by an increase in ANC (Absolute Neutrophil Count) of more than $1 \times 10^{9} / \mathrm{L}[3,4]$. These patients benefit greatly from G-CSF $[5,4]$; such as a significant improvement in the quality of life, including health, performance in society and socioeconomic status, reduction in the frequency and severity of infections, fever, use of antibiotics, hospitalization, oral ulcers and severity, and increased survival $[6-9,5,10-12]$. Also, treating children with severe congenital neutropenia severely reduces the risk of sepsis [13]. This cytokine significantly improves the quality of life of patients. Treatment with rhG-CSF improves all previous chronic infections, decreases the frequency of new episodes of infection, and discontinues the administration of prophylactic antibiotics [6]. Various studies have been conducted on the side effects of G-CSF. Increased spleen size has been reported in most patients. Also, the effect of G-CSF on bone marrow stimulation and development appears as early bone pain [8]. According to the SCNIR, side effects of these patients include bone pain, splenomegaly, thrombocytopenia, osteoporosis, and leukemia/MDS [14, 4] and also fever, myalgia, and erythema [15]. G-CSF has several effects on the granulocytic cell line. Not only does it stimulate the growth and differentiation of myeloid precursors, but it also enhances the activity of adult neutrophils [16]. According to numerous studies, side effects of this drug include splenomegaly, thrombocytopenia, osteopenia and osteoporosis, bone pain, vasculitis, skin rash, eosinophilia, monocytosis and malignant changes to AML/MDS [7, 2, 17, 18, 3, 19, 10, 4]. In other disparate studies, hyperplasia, glomerulonephritis, myalgia, erythema, dyspnea, hypotension, sweating, and hot flashes have also been observed $[20,15,2]$. The most important of these complications is the advancement of AML / MDS, which is still 
unclear whether G-CSF is the cause of this transformation or because of the inherent tendency of the congenital neutropenic disease to progress to MDS / AML, the increased survival of congenital patients by G-CSF Provides a good opportunity to develop this malignancy [21,22]. Cytogenetic abnormalities have been reported with a high risk of these malignancies: CSF3R mutation (G-CSF receptor), ELA2 gene mutation, Rascogenic activity, chromosome 7 monosomal, and chromosomal changes that in studies conducted in a number of patients, resistance to G-CSF therapy and the continuation of severe infections that are often life-threatening are reported, with HSCT, is the only treatment available $[23,3,24]$.

As already stated PDGRASTIM or Filgrastim (PDG) and PDLASTA or Peg-Filgrastim (PDL) had been a comparison in a head to head trial with brand one (GCSF and Neulasta) and show non-inferiority results. So most of the patients should take some kinds of GCSF during dense dose treatment if the treatment is GCSF, the patient should come to the center every day after chemo for at least 6 days, but if they take PDL just one shoot required, so the objective of this study is to compare the drug effect and side effects between two drugs in breast cancer patients in a non-inferiority clinical trial.

\section{Methods}

This interventional study compared the efficacy and safety of the PDL product of Pooyesh Darou Biopharmaceuticals Company with PDG in breast cancer patients as a non-inferiority parallel groups Randomized Clinical Trial. The flow diagram has been demonstrated in Fig. 1.

\section{Patients and criteria}

This study included 80 patients diagnosed with breast cancer. They were treated with adjuvant and neoadjuvant therapy with a dose-dense AC*4-T*4 regimen which consists of four courses of Adriamicine plus Cyclophosphamide and four courses of Taxen based drugs. They referred to Motamed Cancer Institute for Chemotherapy.

\section{Inclusion criteria were:}

- Age $>18$ years

- Investigator diagnosis of breast cancer candidate of adjuvant therapy

- Absolute neutrophil count $\geq 1.5 \times 10^{9} / \mathrm{I}$

- platelet count $\geq 100 \times 10^{9} / /$

- Serum creatinine $<1.5 \times$ upper limits of normal

- indication of receiving GCSF

\section{Exclusion criteria consisted of:}

- Bilirubin > upper limit of normal; or aspartate transaminase and/or alanine transaminase $>1.5 \times$ upper limits of normal, concomitant with alkaline phosphatase $>2.5 \times$ upper limit of normal

- Radiation therapy within 4 weeks of randomization into this study

- Prior bone marrow or stem cell transplantation

- Total lifetime exposure to doxorubicin $>240 \mathrm{mg} / \mathrm{m}^{2}$ or epirubicin $>600 \mathrm{mg} / \mathrm{m}^{2}$

- Ejection Fraction $<40 \%$ 
In case of any serious complications due to the use of PDL, the patient was excluded from the study.

In this study, breast cancer patients who were under adjuvant and neo_adjuvant chemotherapy with a dose-dense $A C \star 4-T * 4$ regimen, considering inclusion and exclusion criteria, signed informed consent and recruited the study. They were randomly assigned to the drug group (PDL) or to the control group (PDG) and demographic and clinical characteristics were recorded. Those drugs were prescribed free of charge and were injected with the supervision of a doctor at the treatment center. Patients in the two groups received up to 8 courses of chemotherapy, which the first 4 courses consisted of Adriamycin and the second half of treatment courses consisted of taxen based drugs. Hematologic parameters and the possible side effects of the drug were recorded according to Cell Blood Count and patients' symptoms on start day and 7th and 15th days of each chemotherapy course.

The incidence of Febrile Neutropenia in each cycle was assumed as a primary efficacy outcome. according to Holmes's study[25], the rate of success in PDL and PDG in a lower incidence of neutropenia was 0.91 and 0.82 , respectively. Considering the Non-inferiority margin of 0.1 , the Allocation ratio of $1 / 1$, a error equal to 0.05 , and $80 \%$ power of the study, 40 patients were recruited to the study.

The patients were trained to notify any complications by telephone to the design interface who was presented in the consent form. If a patient had fever and neutropenia in the first week or the second week after treatment, at least three additional doses with appropriate antibiotics were prescribed and, if the physician advised, she was admitted to the hospital. In patients with Grade 3 or 4 of neutropenia, PDL would change to PDG up to the end of treatment. Those patients were excluded from the PDL group. If the patient was admitted, all drug costs were paid by the Pharmaceutical Drug Company.

\section{Intervention:}

Patients in the PDL group received a single subcutaneous injection of $6 \mathrm{mg}$ Peg-Filgrastim on the second day of each chemotherapy cycle. In the PDG group, in each chemotherapy cycle, 300 micrograms Filgrastim per day was injected subcutaneously for six consecutive days.

\section{Outcome}

The outcome of interests were hematologic parameters consisting of WBC, Hgb, Platelet, Neutrophil, Lymphocyte, and ANC which their values were compared between two groups in each course and during eight courses of chemotherapy. ANC was calculated by multiplying the percentage of neutrophils by the total number of WBCs (in thousands). The short and long term side effects of the drugs were recorded in both groups during the study.

\section{Randomization and blinding}

Randomization was performed using quadruple blocks. Blocks of random allocation consisted of pockets provided by corresponding researcher. Concealment was supervised by one the clinic personnel who was not involve in enrollment of patients. The oncologist assigned participants to interventions. Due to the different protocols of administration of the two drugs and the need for supervision of an oncologist, blinding of the patient and the therapist was not possible. The statistical analyzer was not informed about the assignment of patients to the groups.

\section{Statistical analysis}

An Interim analysis was achieved after completing one third of sample size. Since no side effect was noticed in two groups, the recruitment was continued up to the end of study. The frequency of demographic and clinical characteristics of the two groups was demonstrated by descriptive statistics. The randomized allocation of two groups 
of the study was assessed by chi-square and t-student tests. Kolmogorov-Smirnov test was applied to evaluate the normality of outcome variables' distribution. Most variables did not show the normal distribution and nonparametric tests were applied in the next steps of analysis.

The mean and median of hematologic variables (WBC, HgB, platelet, neutrophil, lymphocyte, and ANC) distribution and frequency of complications were compared in PDG and PDL groups.

The changes in hematologic variables (WBC, $\mathrm{HgB}$, platelet, neutrophil, lymphocyte and ANC) during start point, 7th and 15th days in each course of chemotherapy were evaluated in both groups by Friedman analysis. This variation of repeated measurements of outcome between two groups of the study was compared by Generalized Estimation Equation (GEE) analysis. Hematologic parameters' changes during 8 courses of chemotherapy, were compared between two groups by Generalized Estimation Equation analysis too. Statistical analysis was performed by SPSS software version 22.

\section{Ethical considerations}

Patients entered the study by signing a written informed consent for drug intake. All information such as emphasizing the process of implementation, right of exclusion of study during the treatment, covering expenses, possible side effects and emergency phone number for consultation and reporting side effects had been included in the informed consent form. This research was approved and registered in the Ethics Committee of Breast Cancer Research Center with code number: IR.ACECR.IBCRC.REC.1395.19. Also, this study was registered in the Iranian Registry of Clinical Trials (IRCT) in https://www.irct.ir/ with registration code: IRCT20190504043465N1.

\section{Results}

Eighty patients were recruited to PDL and PDG groups equally. Table 1 shows the comparison of demographic and clinical characteristics of breast cancer patients treated by PDG or PDL. The mean age of PDG and PDL groups were $47.8 \pm 9.04$ and $43.7 \pm 9.23$, respectively. There was no significant difference between two groups in terms of age, BMI, tumor size, excised LN, involved LN, Ki-67 index, education, marital, employment, ER, PR, and HER2 status. 
Table 1

Demographic and clinical characteristics of patients in two study groups

\begin{tabular}{|c|c|c|c|}
\hline Variable & PDG & PDL & P-value \\
\hline & Mean \pm SD & Mean \pm SD & \\
\hline Age, year & $47.8 \pm 9.04$ & $43.7 \pm 9.23$ & 0.05 \\
\hline BMI, kg/m² & $27.8 \pm 4.88$ & $26.6 \pm 4.06$ & 0.261 \\
\hline Tumor size, cm & $3.0 \pm 1.1$ & $3.1 \pm 0.9$ & 0.744 \\
\hline Excised LN, n & $7.9 \pm 4.6$ & $8.7 \pm 6.1$ & 0.566 \\
\hline Involved LN, n & $2.1 \pm 2.7$ & $2.1 \pm 2.6$ & 0.958 \\
\hline \multirow[t]{2}{*}{$\mathrm{Ki}-67, \%$} & $36.0 \pm 23.2$ & $34.6 \pm 26.5$ & 0.819 \\
\hline & No (\%) & No (\%) & \\
\hline Age & & & 0.116 \\
\hline$<50$ & $24(60)$ & $30(75)$ & \\
\hline$\geq 50$ & $16(40)$ & $10(25)$ & \\
\hline Education status & & & 0.362 \\
\hline Illiterate / Elementary & $16(42.1)$ & $19(48.7)$ & \\
\hline Diploma / Academic & $22(57.9)$ & $20(51.3)$ & \\
\hline Marital status & & & 0.387 \\
\hline Married & $33(82.5)$ & $33(87.5)$ & \\
\hline Single / Divorce / Widow & $7(17.5)$ & $5(12.5)$ & \\
\hline Employment status & & & 0.293 \\
\hline Housewife & $30(75.5)$ & $33(82.5)$ & \\
\hline Employed & $10(25)$ & $7(17.5)$ & \\
\hline ER & & & 0.150 \\
\hline Negative & $11(28.2)$ & $6(15.8)$ & \\
\hline Positive & $28(71.8)$ & $32(84.2)$ & \\
\hline PR & & & 0.061 \\
\hline Negative & $19(48.7)$ & $11(28.9)$ & \\
\hline Positive & $20(51.3)$ & $27(71.1)$ & \\
\hline HER2 & & & 0.587 \\
\hline Negative & $31(79.5)$ & 30 (78.9) & \\
\hline Positive & $8(20.5)$ & $8(21.1)$ & \\
\hline
\end{tabular}


Outcome measurements were achieved at the start point, 7th and 15th days in each course of chemotherapy. The distribution of hematologic variables in eight courses of chemotherapy in both groups of study has been demonstrated in Table 2. 
Table 2

Changes of hematologic variables during eight courses of chemotherapy in two groups

\begin{tabular}{|c|c|c|c|c|c|c|c|}
\hline Time & variable & $\begin{array}{l}\text { PDG } \\
\text { Mean } \pm \text { SD }\end{array}$ & $\begin{array}{l}\text { PDL } \\
\text { Mean } \pm \text { SD }\end{array}$ & Time & variable & $\begin{array}{l}\text { PDG } \\
\text { Mean } \pm \text { SD }\end{array}$ & $\begin{array}{l}\text { PDL } \\
\text { Mean } \pm \text { SD }\end{array}$ \\
\hline \multirow{6}{*}{$\begin{array}{l}\text { Start of } \\
\text { study }\end{array}$} & WBC & $\begin{array}{l}9068.42 \pm \\
1433.59\end{array}$ & $\begin{array}{l}8361.58 \pm \\
1992.03\end{array}$ & \multirow{6}{*}{$\begin{array}{l}5_{\text {th }} \\
\text { course } \\
\text { (7th day) }\end{array}$} & \multirow{3}{*}{$\begin{array}{l}\text { WBC } \\
\text { Hgb } \\
\text { Platelet } \\
\text { Neutrophil }\end{array}$} & $\begin{array}{l}2593.42 \pm \\
538.79\end{array}$ & $\begin{array}{l}2455.52 \pm \\
688.89\end{array}$ \\
\hline & $\mathrm{Hgb}$ & $12.75 \pm 1.04$ & $12.98 \pm 1.2$ & & & $11.78 \pm .93$ & $11.93 \pm .79$ \\
\hline & \multirow{4}{*}{$\begin{array}{l}\text { Platelet } \\
\text { Neutrophil } \\
\text { Lymphocyte } \\
\text { ANC }\end{array}$} & $\begin{array}{l}298972.22 \\
\pm \\
130889.81\end{array}$ & $\begin{array}{l}281910.53 \\
\pm \\
104249.08\end{array}$ & & & $\begin{array}{l}104027.78 \\
\pm 12112.53\end{array}$ & $\begin{array}{l}100473.68 \\
\pm 29235.82\end{array}$ \\
\hline & & $74.5 \pm 6.62$ & $75.37 \pm 6.09$ & & \multirow{3}{*}{ ANC } & $\begin{array}{l}50.53 \pm \\
6.55\end{array}$ & $\begin{array}{l}52.63 \pm \\
5.54\end{array}$ \\
\hline & & & $22.92 \pm 6.92$ & & & $\begin{array}{l}47.79 \pm \\
9.516\end{array}$ & $\begin{array}{l}46.55 \pm \\
6.41\end{array}$ \\
\hline & & $6659 \pm 1263$ & $6388 \pm 1682$ & & & $1312 \pm 360$ & $1299 \pm 406$ \\
\hline \multirow{7}{*}{$\begin{array}{l}1_{\text {st }} \text { course } \\
\text { (7th day) }\end{array}$} & \multirow{2}{*}{$\begin{array}{l}\text { WBC } \\
\mathrm{Hgb}\end{array}$} & $\begin{array}{l}3623.68 \pm \\
1272.46\end{array}$ & $\begin{array}{l}5997 \pm \\
12404\end{array}$ & \multirow{7}{*}{$\begin{array}{l}5_{\text {th }} \\
\text { course } \\
\text { (15th } \\
\text { day) }\end{array}$} & \multirow{3}{*}{$\begin{array}{l}\text { WBC } \\
\text { Hgb } \\
\text { Platelet } \\
\text { Neutrophil }\end{array}$} & $\begin{array}{l}5339.47 \pm \\
1450.54\end{array}$ & $\begin{array}{l}5121.08 \pm \\
1699.03\end{array}$ \\
\hline & & $12.56 \pm 1.01$ & $12.8 \pm 1.01$ & & & $11.68 \pm .96$ & $11.89 \pm .76$ \\
\hline & $\begin{array}{l}\text { Platelet } \\
\text { Neutrophil }\end{array}$ & $\begin{array}{l}141722.22 \\
\pm 77288.18\end{array}$ & $\begin{array}{l}136584 \pm \\
32865\end{array}$ & & & $\begin{array}{l}153888.89 \\
\pm 21372.58\end{array}$ & $\begin{array}{l}158657.89 \\
\pm 42731.9\end{array}$ \\
\hline & \multirow{4}{*}{$\begin{array}{l}\text { Lymphocyte } \\
\text { ANC }\end{array}$} & $46.5 \pm 6.19$ & $47.6 \pm 6.3$ & & \multirow{4}{*}{$\begin{array}{l}\text { Lymphocyte } \\
\text { ANC }\end{array}$} & \multirow[t]{2}{*}{$\begin{array}{l}88.24 \pm \\
6.32\end{array}$} & $\begin{array}{l}88.58 \pm \\
4.18\end{array}$ \\
\hline & & $52.97 \pm 6.57$ & $51.3 \pm 6.6$ & & & & \multirow{3}{*}{$\begin{array}{l}10.87 \pm 3.4 \\
4554 \pm \\
1597\end{array}$} \\
\hline & & $1662 \pm 737$ & $2896 \pm 6062$ & & & $\begin{array}{l}11.63 \pm \\
6.41\end{array}$ & \\
\hline & & & & & & $\begin{array}{l}4681 \pm \\
1328\end{array}$ & \\
\hline \multirow{7}{*}{$\begin{array}{l}1_{\text {st }} \text { course } \\
\text { (15th } \\
\text { day) }\end{array}$} & \multirow{2}{*}{$\begin{array}{l}\text { WBC } \\
\mathrm{Hgb}\end{array}$} & $\begin{array}{l}5618.42 \pm \\
1216.74\end{array}$ & $\begin{array}{l}5827.63 \pm \\
1127.22\end{array}$ & \multirow{7}{*}{$\begin{array}{l}6_{\text {th }} \\
\text { course } \\
\text { (7th day) }\end{array}$} & \multirow{3}{*}{$\begin{array}{l}\text { WBC } \\
\text { Hgb } \\
\text { Platelet } \\
\text { Neutrophil }\end{array}$} & $\begin{array}{l}3515.79 \pm \\
701.65\end{array}$ & $\begin{array}{l}3531.58 \pm \\
889.23\end{array}$ \\
\hline & & $12.52 \pm .95$ & $12.76 \pm$ & & & $11.6 \pm 1.03$ & $11.85 \pm .71$ \\
\hline & $\begin{array}{l}\text { Platelet } \\
\text { Neutrophil }\end{array}$ & $\begin{array}{l}182694.44 \\
\pm 35740.19\end{array}$ & $\begin{array}{l}185578.95 \\
\pm 32961.59\end{array}$ & & & $\begin{array}{l}118111.11 \\
\pm 22193.66\end{array}$ & $\begin{array}{l}116078.95 \\
\pm 17653.4\end{array}$ \\
\hline & Lymphocyte & $85.18 \pm 5.4$ & $84.66 \pm 5.54$ & & Lymphocyte & $\begin{array}{l}51.66 \pm \\
6.84\end{array}$ & $52.6 \pm 6.82$ \\
\hline & ANC & $14.45 \pm 4.75$ & $15+5.57$ & & ANC & & $\begin{array}{l}46.95 \pm \\
7.17\end{array}$ \\
\hline & & $4655 \pm 1292$ & & & & 7.03 & \\
\hline & & & טוֹ ותשד & & & $1809 \pm 460$ & טעם \\
\hline
\end{tabular}




\begin{tabular}{|c|c|c|c|c|c|c|c|}
\hline Time & variable & $\begin{array}{l}\text { PDG } \\
\text { Mean } \pm \text { SD }\end{array}$ & $\begin{array}{l}\text { PDL } \\
\text { Mean } \pm \text { SD }\end{array}$ & Time & variable & $\begin{array}{l}\text { PDG } \\
\text { Mean } \pm \text { SD }\end{array}$ & $\begin{array}{l}\text { PDL } \\
\text { Mean } \pm \text { SD }\end{array}$ \\
\hline $\begin{array}{l}2_{\text {nd }} \\
\text { course } \\
\text { (7th day) }\end{array}$ & $\begin{array}{l}\text { WBC } \\
\text { Hgb } \\
\text { Platelet } \\
\text { Neutrophil } \\
\text { Lymphocyte } \\
\text { ANC }\end{array}$ & $\begin{array}{l}2786.84 \pm \\
850.17 \\
12.36 \pm 1.03 \\
109055.55 \\
\pm 19608.95 \\
47.71 \pm 6.21 \\
51.23 \pm 6.09 \\
1324 \pm 476\end{array}$ & $\begin{array}{l}3553.16 \pm \\
3202.25 \\
12.44 \pm 1.06 \\
117118.42 \\
\pm 20749.29 \\
50.52 \pm 8.98 \\
48.21 \pm \\
10.82 \\
1819 \pm 1891\end{array}$ & $\begin{array}{l}\sigma_{\text {th }} \\
\text { course } \\
\text { (15th } \\
\text { day) }\end{array}$ & $\begin{array}{l}\text { WBC } \\
\text { Hgb } \\
\text { Platelet } \\
\text { Neutrophil } \\
\text { Lymphocyte } \\
\text { ANC }\end{array}$ & $\begin{array}{l}7200 \pm \\
1567.23 \\
11.58 \pm .9 \\
173972.22 \\
\pm 19983.54 \\
88.73 \pm \\
5.23 \\
11.13 \pm 5.2 \\
6371 \pm \\
1558\end{array}$ & $\begin{array}{l}7056.58 \pm \\
1948.95 \\
11.76 \pm .72 \\
171236.84 \\
\pm 37220.62 \\
88.37 \pm \\
3.97 \\
11.63 \pm \\
3.97 \\
6244 \pm \\
1792\end{array}$ \\
\hline $\begin{array}{l}2_{\text {nd }} \\
\text { course } \\
\text { (15th } \\
\text { day) }\end{array}$ & $\begin{array}{l}\text { WBC } \\
\text { Hgb } \\
\text { Platelet } \\
\text { Neutrophil } \\
\text { Lymphocyte } \\
\text { ANC }\end{array}$ & $\begin{array}{l}5405.52 \pm \\
5803.10 \\
12.25 \pm .96 \\
152444.44 \\
\pm 12622.98 \\
86.73 \pm 5.75 \\
14.02 \pm 8.14 \\
4550 \pm 4351\end{array}$ & $\begin{array}{l}4693.42 \pm \\
831.65 \\
12.4 \pm 1.18 \\
160131.58 \\
\pm 20145.64 \\
86.34 \pm 4.39 \\
12.89 \pm 3.67 \\
4009 \pm 808\end{array}$ & $\begin{array}{l}7_{\text {th }} \\
\text { course } \\
\text { (7th day) }\end{array}$ & $\begin{array}{l}\text { WBC } \\
\text { Hgb } \\
\text { Platelet } \\
\text { Neutrophil } \\
\text { Lymphocyte } \\
\text { ANC }\end{array}$ & $\begin{array}{l}5722.37 \pm \\
7130.64 \\
11.52 \pm .88 \\
124611.11 \\
\pm 15331.16 \\
52.87 \pm 5.5 \\
46.34 \pm \\
5.82 \\
3086 \pm \\
4284\end{array}$ & $\begin{array}{l}4190.79 \pm \\
757.38 \\
11.69 \pm .73 \\
122815.79 \\
\pm 16204.53 \\
68.34 \pm \\
98.78 \\
46.18 \pm \\
8.47 \\
2923 \pm \\
4491\end{array}$ \\
\hline $\begin{array}{l}3_{\text {rd }} \\
\text { course } \\
\text { (7th day) }\end{array}$ & $\begin{array}{l}\text { WBC } \\
\text { Hgb } \\
\text { Platelet } \\
\text { Neutrophil } \\
\text { Lymphocyte } \\
\text { ANC }\end{array}$ & $\begin{array}{l}2610.52 \pm \\
1345.12 \\
12.14 \pm .93 \\
97802.78 \pm \\
14290.07 \\
47.4 \pm 6.9 \\
51.97 \pm 6.52 \\
1215 \pm 582\end{array}$ & $\begin{array}{l}2660.52 \pm \\
1277.09 \\
15.23 \pm \\
17.99 \\
103476.31 \\
\pm \\
132901.69 \\
51.18 \pm 4.57 \\
47.55 \pm 5.25 \\
1359 \pm 636\end{array}$ & $\begin{array}{l}7_{\text {th }} \\
\text { course } \\
\text { (15th } \\
\text { day) }\end{array}$ & $\begin{array}{l}\text { WBC } \\
\text { Hgb } \\
\text { Platelet } \\
\text { Neutrophil } \\
\text { Lymphocyte } \\
\text { ANC }\end{array}$ & $\begin{array}{l}11797.37 \\
\pm 14999.43 \\
11.48 \pm .95 \\
183500 \pm \\
21285.14 \\
89.44 \pm \\
4.85 \\
9.05 \pm 3.38 \\
10515 \pm \\
13354\end{array}$ & $\begin{array}{l}8007.9 \pm \\
2431.03 \\
11.63 \pm .76 \\
181289.47 \\
\pm 31302.28 \\
90.63 \pm \\
3.83 \\
10.64 \pm \\
10.17 \\
7273 \pm \\
2273\end{array}$ \\
\hline
\end{tabular}




\begin{tabular}{|c|c|c|c|c|c|c|c|}
\hline Time & variable & $\begin{array}{l}\text { PDG } \\
\text { Mean } \pm \text { SD }\end{array}$ & $\begin{array}{l}\text { PDL } \\
\text { Mean } \pm \text { SD }\end{array}$ & Time & variable & $\begin{array}{l}\text { PDG } \\
\text { Mean } \pm \text { SD }\end{array}$ & $\begin{array}{l}\text { PDL } \\
\text { Mean } \pm \text { SD }\end{array}$ \\
\hline $\begin{array}{l}3_{\text {rd }} \\
\text { course } \\
\text { (15th } \\
\text { day) }\end{array}$ & $\begin{array}{l}\text { WBC } \\
\text { Hgb } \\
\text { Platelet } \\
\text { Neutrophil } \\
\text { Lymphocyte } \\
\text { ANC }\end{array}$ & $\begin{array}{l}3961.31 \pm \\
668.86 \\
12.06 \pm .92 \\
142861.11 \\
\pm 14204.93 \\
87.1 \pm 5.05 \\
12.81 \pm 5.11 \\
1864 \pm 431\end{array}$ & $\begin{array}{l}3993.68 \pm \\
717.65 \\
15.14 \pm \\
17.99 \\
145589.47 \\
\pm 27305.87 \\
87.87 \pm 4.47 \\
12 \pm 4.6 \\
2010 \pm 450\end{array}$ & $\begin{array}{l}8_{\text {th }} \\
\text { course } \\
\text { (7th day) }\end{array}$ & $\begin{array}{l}\text { WBC } \\
\text { Hgb } \\
\text { Platelet } \\
\text { Neutrophil } \\
\text { Lymphocyte } \\
\text { ANC }\end{array}$ & $\begin{array}{l}5396.05 \pm \\
1612.13 \\
11.15 \pm \\
1.91 \\
128583.33 \\
\pm 20230.63 \\
53.55 \pm \\
6.03 \\
45.39 \pm \\
8.08 \\
2924 \pm \\
1057\end{array}$ & $\begin{array}{l}5010.53 \pm \\
945.41 \\
11.51 \pm .81 \\
127594.74 \\
\pm 17224.53 \\
52.21 \pm \\
7.29 \\
46.63 \pm \\
7.65 \\
2624 \pm 648\end{array}$ \\
\hline $\begin{array}{l}4_{\text {th }} \\
\text { course } \\
\text { (7th day) }\end{array}$ & $\begin{array}{l}\text { WBC } \\
\text { Hgb } \\
\text { Platelet } \\
\text { Neutrophil } \\
\text { Lymphocyte } \\
\text { ANC }\end{array}$ & $\begin{array}{l}2284.47 \pm \\
1369.58 \\
11.92 \pm .92 \\
90383.33 \pm \\
28554.37 \\
49.1 \pm 10.91 \\
50.29 \pm \\
10.68 \\
1148 \pm 881\end{array}$ & $\begin{array}{l}1976.58 \pm \\
380.76 \\
12.07 \pm .84 \\
92842.1 \pm \\
11083.29 \\
50.26 \pm 4.92 \\
47.68 \pm 6.63 \\
3711 \pm 968\end{array}$ & $\begin{array}{l}8_{\text {th }} \\
\text { course } \\
\text { (15th } \\
\text { day) }\end{array}$ & $\begin{array}{l}\text { WBC } \\
\text { Hgb } \\
\text { Platelet } \\
\text { Neutrophil } \\
\text { Lymphocyte } \\
\text { ANC }\end{array}$ & $\begin{array}{l}13300 \pm \\
6704.21 \\
11.52 \pm \\
1.24 \\
193444.44 \\
\pm 26144.04 \\
88.94 \pm \\
7.64 \\
10.68 \pm \\
7.39 \\
11861 \pm \\
6426\end{array}$ & $\begin{array}{l}13448.68 \\
\pm 17942.84 \\
11.41 \pm .87 \\
194526.31 \\
\pm 41315.36 \\
90.05 \pm \\
4.77 \\
9.71 \pm 4.79 \\
12133 \pm \\
16165\end{array}$ \\
\hline $\begin{array}{l}\text { 4th } \\
\text { course } \\
\text { (15th } \\
\text { day) }\end{array}$ & $\begin{array}{l}\text { WBC } \\
\text { Hgb } \\
\text { Platelet } \\
\text { Neutrophil } \\
\text { Lymphocyte } \\
\text { ANC }\end{array}$ & $\begin{array}{l}4213.15 \pm \\
4968.81 \\
11.61 \pm 1.95 \\
131355.55 \\
\pm 27377.83 \\
86.52 \pm 7.29 \\
13.34 \pm 7.21 \\
3563 \pm 3892\end{array}$ & $\begin{array}{l}3415.79 \pm \\
789.98 \\
11.98 \pm .78 \\
134000 \pm \\
27496.44 \\
86.79 \pm 4.89 \\
13.08 \pm 4.74 \\
996 \pm 222\end{array}$ & & & & \\
\hline
\end{tabular}

Figure 2 shows the changes in ANC values during eight courses of chemotherapy in PDG and PDL groups. Similar trends of ANC values are notifiable between two groups.

Within and between groups' variation of blood counts was analyzed during start point, 7th and 15th days in each course of chemotherapy. (Table 3) The results showed that in PDL and PGL groups, all of the hematologic components (WBC, Hgb, Plt, Neut, Lymph and ANC) had significant changes during each course of chemotherapy. Decreasing the hematologic component and increasing after GCF injection in each course is the prominent pattern of data variations. But in the fifth course of PDL injection, Hgb showed no significant change $(P=0.095)$. 
Table 3

Comparing within and between groups blood counts measured at the start point, 7th and 15th days in each course of chemotherapy

\begin{tabular}{|c|c|c|c|c|c|c|c|c|c|c|}
\hline \multirow[t]{2}{*}{ Courses } & & \multicolumn{3}{|c|}{ PDL Group (Median) } & \multirow{2}{*}{$\begin{array}{l}\mathrm{p}- \\
\text { value* }\end{array}$} & \multicolumn{3}{|c|}{ PGL Group (Median) } & \multirow{2}{*}{$\begin{array}{l}\mathrm{p}^{-} \\
\text {value* }^{*}\end{array}$} & \multirow{2}{*}{$\begin{array}{l}\mathrm{p}- \\
\text { value**}\end{array}$} \\
\hline & & DO & D7 & D15 & & DO & D7 & D15 & & \\
\hline \multirow[t]{6}{*}{1} & WBC & 8900 & 4100 & 5850 & $\begin{array}{l}< \\
0.001\end{array}$ & 9550 & 3850 & 5750 & $\begin{array}{l}< \\
0.001\end{array}$ & 0.295 \\
\hline & $\mathrm{Hb}$ & 13.20 & 13.00 & 13.00 & $\begin{array}{l}< \\
0.001\end{array}$ & 12.80 & 12.35 & 12.25 & $\begin{array}{l}< \\
0.001\end{array}$ & 0.159 \\
\hline & Plt & 280000 & 124000 & 173000 & $\begin{array}{l}< \\
0.001\end{array}$ & 248500 & 124000 & 168000 & $\begin{array}{l}< \\
0.001\end{array}$ & 0.416 \\
\hline & Neut & 75 & 50 & 85 & $\begin{array}{l}< \\
0.001\end{array}$ & 70 & 50 & 85 & $\begin{array}{l}< \\
0.001\end{array}$ & 0.312 \\
\hline & Lymph & 25 & 50 & 15 & $\begin{array}{l}< \\
0.001\end{array}$ & 27 & 50 & 15 & $\begin{array}{l}< \\
0.001\end{array}$ & 0.130 \\
\hline & ANC & 6545 & 2050 & 5025 & $\begin{array}{l}< \\
0.001\end{array}$ & 6830 & 1660 & 4600 & $\begin{array}{l}< \\
0.001\end{array}$ & 0.327 \\
\hline \multirow[t]{6}{*}{2} & WBC & 5850 & 3100 & 4400 & $\begin{array}{l}< \\
0.001\end{array}$ & 5750 & 3000 & 4500 & $\begin{array}{l}< \\
0.001\end{array}$ & 0.866 \\
\hline & $\mathrm{Hb}$ & 13.00 & 13.00 & 12.90 & $\begin{array}{l}< \\
0.001\end{array}$ & 12.25 & 12.20 & 12.20 & $\begin{array}{l}< \\
0.001\end{array}$ & 0.307 \\
\hline & Plt & 173000 & 111000 & 156000 & $\begin{array}{l}< \\
0.001\end{array}$ & 168000 & 107000 & 156000 & $\begin{array}{l}< \\
0.001\end{array}$ & 0.468 \\
\hline & Neut & 85 & 50 & 85 & $\begin{array}{l}< \\
0.001\end{array}$ & 85 & 50 & 86 & $\begin{array}{l}< \\
0.001\end{array}$ & 0.504 \\
\hline & Lymph & 15 & 50 & 13 & $\begin{array}{l}< \\
0.001\end{array}$ & 15 & 50 & 14 & $\begin{array}{l}< \\
0.001\end{array}$ & 0.243 \\
\hline & ANC & 5025 & 1575 & 3838 & $\begin{array}{l}<.001 \\
0.001\end{array}$ & 4600 & 1400 & 3895 & $\begin{array}{l}< \\
0.001\end{array}$ & 0.950 \\
\hline \multirow[t]{6}{*}{3} & WBC & 4400 & 2450 & 3950 & $\begin{array}{l}< \\
0.001\end{array}$ & 4500 & 2500 & 3950 & $\begin{array}{l}< \\
0.001\end{array}$ & 0.820 \\
\hline & $\mathrm{Hb}$ & 12.90 & 12.90 & 12.30 & $\begin{array}{l}<.001 \\
0.00\end{array}$ & 12.20 & 12.00 & 12.00 & $\begin{array}{l}< \\
0.001\end{array}$ & 0.107 \\
\hline & Plt & 156000 & 101000 & 139000 & $\begin{array}{l}< \\
0.001\end{array}$ & 156000 & 100000 & 146000 & $\begin{array}{l}< \\
0.001\end{array}$ & 0.302 \\
\hline & Neut & 85 & 50 & 90 & $\begin{array}{l}< \\
0.001\end{array}$ & 86 & 50 & 90 & $\begin{array}{l}< \\
0.001\end{array}$ & 0.023 \\
\hline & Lymph & 15 & 50 & 10 & $\begin{array}{l}<.001 \\
0.01\end{array}$ & 14 & 50 & 10 & $\begin{array}{l}< \\
0.001\end{array}$ & 0.006 \\
\hline & ANC & 3838 & 1305 & 1975 & $\begin{array}{l}< \\
0.001\end{array}$ & 3895 & 1200 & 1900 & $\begin{array}{l}< \\
0.001\end{array}$ & 0.229 \\
\hline
\end{tabular}

p-value*: Repeated measurements within groups (Friedman test)

p-value**: Repeated measurements Between groups (GEE analysis) 


\begin{tabular}{|c|c|c|c|c|c|c|c|c|c|c|}
\hline \multirow[t]{6}{*}{4} & WBC & 3950 & 1900 & 3100 & $<_{0.001}^{<}$ & 3950 & 2100 & 3400 & 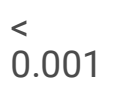 & 0.443 \\
\hline & $\mathrm{Hb}$ & 12.30 & 12.05 & 12.05 & $\begin{array}{c}< \\
0.001\end{array}$ & 12.00 & 12.00 & 12.00 & $\begin{array}{l}< \\
0.001\end{array}$ & 0.473 \\
\hline & Plt & 139000 & 92500 & 128500 & 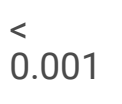 & 146000 & 98000 & 132000 & $<0.001$ & 0.969 \\
\hline & Neut & 90 & 50 & 90 & $\begin{array}{l}< \\
0.001\end{array}$ & 90 & 50 & 90 & $\begin{array}{l}< \\
0.001\end{array}$ & 0.317 \\
\hline & Lymph & 10 & 50 & 11 & $\begin{array}{l}< \\
0.001\end{array}$ & 10 & 50 & 10 & $\begin{array}{l}< \\
0.001\end{array}$ & 0.143 \\
\hline & ANC & 1975 & 955 & 2738 & $\begin{array}{l}< \\
0.001\end{array}$ & 1900 & 1020 & 2872 & $\begin{array}{l}< \\
0.001\end{array}$ & 0.548 \\
\hline \multirow[t]{6}{*}{5} & WBC & 3100 & 2500 & 4500 & $\begin{array}{c}< \\
0.001\end{array}$ & 3400 & 2500 & 4900 & $\begin{array}{c}< \\
0.001\end{array}$ & 0.341 \\
\hline & $\mathrm{Hb}$ & 12.05 & 12.00 & 12.00 & 0.095 & 12.00 & 12.00 & 11.50 & $\begin{array}{c}< \\
0.001\end{array}$ & 0.237 \\
\hline & Plt & 128500 & 100000 & 151000 & $<_{0.001}$ & 132000 & 102000 & 159000 & $<_{0.001}^{<}$ & 0.974 \\
\hline & Neut & 90 & 50 & 90 & $\begin{array}{l}< \\
0.001\end{array}$ & 90 & 50 & 90 & $\overleftarrow{0}_{0.001}$ & 0.413 \\
\hline & Lymph & 11 & 50 & 10 & $\begin{array}{l}< \\
0.001\end{array}$ & 10 & 50 & 10 & $\begin{array}{l}< \\
0.001\end{array}$ & 0.590 \\
\hline & ANC & 2738 & 1350 & 3895 & $\begin{array}{c}< \\
0.001\end{array}$ & 2872 & 1250 & 4410 & $\begin{array}{l}< \\
0.001\end{array}$ & 0.289 \\
\hline \multirow[t]{6}{*}{6} & WBC & 4500 & 3200 & 6750 & $\begin{array}{l}< \\
0.001\end{array}$ & 4900 & 3450 & 6800 & $\begin{array}{l}< \\
0.001\end{array}$ & 0.581 \\
\hline & $\mathrm{Hb}$ & 12.00 & 12.00 & 12.00 & 0.001 & 11.50 & 11.40 & 11.30 & $<0.001$ & 0.206 \\
\hline & Plt & 151000 & 111500 & 159500 & $\begin{array}{l}< \\
0.001\end{array}$ & 159000 & 110000 & 174000 & $\begin{array}{l}< \\
0.001\end{array}$ & 0.919 \\
\hline & Neut & 90 & 50 & 90 & $\iota_{0.001}^{<}$ & 90 & 50 & 90 & $\begin{array}{c}< \\
0.001\end{array}$ & 0.663 \\
\hline & Lymph & 10 & 50 & 10 & $<_{0.001}$ & 10 & 50 & 10 & $\begin{array}{c}< \\
0.001\end{array}$ & 0.743 \\
\hline & ANC & 3895 & 1725 & 5948 & $\begin{array}{l}< \\
0.001\end{array}$ & 4410 & 1900 & 6120 & $\begin{array}{l}< \\
0.001\end{array}$ & 0.543 \\
\hline \multirow[t]{2}{*}{7} & WBC & 6750 & 4200 & 8400 & $<_{0.001}^{<}$ & 6800 & 4600 & 9100 & $\begin{array}{l}< \\
0.001\end{array}$ & 0.050 \\
\hline & $\mathrm{Hb}$ & 12.00 & 11.90 & 11.50 & $\begin{array}{l}< \\
0.001\end{array}$ & 11.30 & 11.30 & 11.30 & 0.002 & 0.342 \\
\hline
\end{tabular}

p-value*: Repeated measurements within groups (Friedman test)

$p$-value**: Repeated measurements Between groups (GEE analysis) 


\begin{tabular}{|c|c|c|c|c|c|c|c|c|c|c|}
\hline & Plt & 159500 & 121000 & 170500 & $\begin{array}{l}< \\
0.001\end{array}$ & 174000 & 124000 & 186000 & $\begin{array}{l}< \\
0.001\end{array}$ & 0.559 \\
\hline & Neut & 90 & 50 & 90 & $\begin{array}{l}< \\
0.001\end{array}$ & 90 & 50 & 90 & $\stackrel{<}{0.001}^{<}$ & 0.306 \\
\hline & Lymph & 10 & 50 & 10 & $\begin{array}{l}< \\
0.001\end{array}$ & 10 & 50 & 10 & $\begin{array}{l}< \\
0.001\end{array}$ & 0.898 \\
\hline & ANC & 5948 & 2100 & 7560 & $\hat{0}_{0.001}$ & 6120 & 2300 & 8330 & $\hat{0}_{0.001}$ & 0.146 \\
\hline \multirow[t]{6}{*}{8} & WBC & 8400 & 4950 & 10000 & $\begin{array}{l}< \\
0.001\end{array}$ & 9100 & 5100 & 10550 & $\hat{0}_{0.001}$ & 0.395 \\
\hline & $\mathrm{Hb}$ & 11.50 & 11.35 & 11.35 & $\begin{array}{l}< \\
0.001\end{array}$ & 11.30 & 11.25 & 11.25 & $\dot{c}_{0.001}$ & 0.528 \\
\hline & Plt & 170500 & 124000 & 186500 & $\begin{array}{l}< \\
0.001\end{array}$ & 186000 & 124000 & 194500 & $\begin{array}{l}< \\
0.001\end{array}$ & 0.637 \\
\hline & Neut & 90 & 50 & 90 & $\begin{array}{l}< \\
0.001\end{array}$ & 90 & 50 & 90 & $\begin{array}{l}< \\
0.001\end{array}$ & 0.781 \\
\hline & Lymph & 10 & 50 & 10 & $<_{0.001}$ & 10 & 50 & 10 & $\begin{array}{l}< \\
0.001\end{array}$ & 0.838 \\
\hline & ANC & 7560 & 2525 & 9000 & $\begin{array}{c}< \\
0.001\end{array}$ & 8330 & 2575 & 9601 & $\begin{array}{l}< \\
0.001\end{array}$ & 0.434 \\
\hline
\end{tabular}

Applying GEE analysis showed no significant difference between the trend of hematologic values during the most courses of chemotherapy. There were two exceptions in the third course of chemotherapy regarding Neutrophil count ( $p=0.023)$ and Lymphocyte count $(p=0.006)$ with lower fluctuations in the PDL group.

Hematologic parameters' changes during 8 courses of chemotherapy, were compared between two groups by Generalized Estimation Equation analysis. The comparison of the WBC ( $p=0.527), \operatorname{Hgb}(p=0.075)$, Platelet $(p=0.819)$, Neutrophil $(p=0.575)$, Lymphocyte $(p=705)$ and ANC $(p=0.675)$ changes during eight courses of treatment identified no statistically significant difference between two study groups.

Since the probability of neutropenia frequency fluctuates during treatment, the changes in blood parameters were compared between the first four courses of chemotherapy with the second four courses. In the PDG group, the mean value of WBC, PIt, and ANC in the first half of treatment were 6281, 153171, and 3358 and in the second half of 6280 , 151064 and 5220, respectively. P-Value of WBC, PIt and ANC the mean difference between two halves was $<0.001$, 0.543 and $<0.001$. In the PDL group, the mean value of WBC, Plt, and ANC in the first half of the treatment were 4446, 154095 and 3249 and in the second half of 6820,152298 and 5575, respectively. P-Value of the WBC, PIt and ANC mean difference between two halves was $<0.001,0.651$ and $<0.001$.

Results showed that WBC ( $p=0.439), \operatorname{Hgb}(p=0.052)$, Platelet $(p=0.7)$, Neutrophil $(p=0.324)$, Lymphocyte $(p=0.463)$ and ANC ( $p=0.571)$ changes during two halves of treatment were not statistically significant between the two study groups.

The comparison of side effects between the two groups is shown in Table 4 . There was no side effect in $50 \%$ of patients of the PDL group compared to $12.5 \%$ in the PDG group. The most common side effects in the PDG group were 
musculoskeletal pain with a relative frequency of $47.5 \%$ compared to $15 \%$ in PDL groups. Headache (30\%), injection site reaction (25\%), leukocytosis (20\%), and bone pain (17.5\%) were other common side effects in the PDG group.

Table 4

Comparison of very common side effects between two groups

\begin{tabular}{|llll|}
\hline Side effect & PDG & PDL & Total \\
& $\mathbf{N}(\%)$ & $\mathbf{N}(\%)$ & $\mathbf{N}(\%)$ \\
\hline No & $5(12.5)$ & $20(50 \%)$ & $25(31.25)$ \\
\hline Headache & $24(30)$ & $10(25)$ & $34(42.5)$ \\
\hline Bone pain & $7(17.5)$ & $3(7.5)$ & $10(12.5)$ \\
\hline Nausea & $3(7.5)$ & $4(10)$ & $7(8.75)$ \\
\hline Musculoskeletal pain & $19(47.5)$ & $6(15)$ & $25(31.25)$ \\
\hline Fever & - & $1(2.5)$ & $1(1.25)$ \\
\hline Injection site reaction & $10(25)$ & $1(2.5)$ & $11(13.75)$ \\
\hline Leukocytosis & $8(20)$ & $4(10)$ & $12(15)$ \\
\hline Non cardiac chest pain & $1(2.5)$ & - & $1(1.25)$ \\
\hline Anaphylaxis & $1(2.5)$ & - & $1(1.25)$ \\
\hline
\end{tabular}

Three patients were excluded from the study. In the PDL group, two patients were excluded from the study because of fever and neutropenia. The first patient received PDL in two courses of chemotherapy. In the third course, ANC on the 7th day of injection decreased to 490. In the second case of this group, an ANC of 750 was recorded at the end of the third course of chemotherapy. Both patients received antibiotics out patiently, and PDL was not continued. In PDG group one patient was hospitalized because of fever and neutropenia after one course of injection. Her ANC on first course of chemotherapy decreased from 5440 to 70 during 15 days of injection. She had oral mucositis and high grade of fever. After three days of antibiotic therapy in hospital, she was recovered.

\section{Discussion}

All of the chemotherapy regimens which were used in this study are those chemotherapy drugs that cause more than $10 \%$ neutropenia without GCF. The chemotherapeutic agents which were used in the study had eight courses. The first four courses are completely similar and composed of Doxorubicin, Cyclophosphamide which cause more neutropenia and fever. But the next four courses consisted of just Docetaxol which cause neutropenia much less than the previous courses. The results showed that WBC $(p=0.439), \mathrm{Hgb}(p=0.052)$, Platelet $(p=0.7)$, Neutrophil $(p=0.324)$, Lymphocyte $(p=0.463)$ and ANC ( $p=0.571)$ changes during two halves of treatment were not statistically significant between the two study groups. Hematologic parameters' changes during the eight courses of chemotherapy identified no statistically significant difference between the two study groups.

In several studies, the efficacy of the PDG drug has been proven. Due to the number of injections administered daily, the drug was slowly released. The Pegylated form of this drug has been effective and safe in clinical trials. Pegylation of drugs improves the clinical level of drugs, such as increased solubility [26] protects the enzyme degradation drug [27], decreases renal clearance [28], physical and thermal stability [29], the longer half-life of antigenicity and toxicity [30]. PDL is a G-CSF quadrilateral conjugate formulation whose efficacy and efficacy are comparable to PDG [31-33]. The half-life of PDL is 12 times longer than the half-life of non-conjunctive drugs. Polyethylene glycol binding to G-CSF 
reduces renal secretion and prevents its proteolysis, resulting in an increase in drug levels up to 14 days after singledose administration. Following the usual chemotherapy, the number of leukocytes and the appearance of CD34 in the peripheral blood after PDL occur faster and sooner than G-CSF [34]. PDG has been used in chemotherapy-induced neutropenic patients and has recently been used in children's neutropenia [32]. In a study by Holmes et al. in 2002, 154 female breast cancer patients were enrolled in the study, 129 of whom received PDL, and 25 received G-CSF. Five patients had unbearable side effects that resulted in discontinuation of the drug, one of these patients suffering from renal insufficiency at a dose of 100 micrograms per kilogram and four others with a dose of at least 30 micrograms had the following side effects: fever, diarrhea, nausea and dehydration. Other side effects seen in all patients were mild to moderate bone pain that was similar to PDL and G-CSF (35\%) and 7\% of patients needed to use narcotic to relieve their pain [24]. There was no side effect in 50\% patients of PDL group compared with $12.5 \%$ in PDG group. Also, the most common side effects in PDG group was musculoskeletal pain with $47.5 \%$ compared to $15 \%$ in PDL groups, wherase, the injection site reaction was the most common side effect with $25 \%$ in PDG group. Headache (30\% vs. $25 \%$ ), injection site reaction ( $25 \%$ vs. $2.5 \%$ ), leukocytosis ( $20 \%$ vs. $10 \%)$, and bone pain $(17.5 \%$ vs. $7.5 \%)$ were other common side effects in PDG group.

In a study of 310 adjuvant chemotherapy patients taking Docetaxel $75 \mathrm{mg}$ daily and Doxorubicin 60 mg per square meter of weight surface area for the first day of each cycle for a maximum of 4 cycles, patients on the second day of the cycle received PDL 100 micrograms per kilogram weight were compared with patients who received PDG 5 micrograms per kilogram weight that the results were comparable in two groups and ANC values in both groups were not significantly different and neutropenia with fever was less common in patients who took PDL. The PDL was tolerated and the side effects profile of the two groups was similar [25]. In another study, which compared the multiple doses of PDL with filgrastim randomly in breast cancer patients, a dose of PDL 100 micrograms per kilogram weight had efficacy and a profile of favorable side effects [24]. In a double-blind, phase III trial with a fixed dose of $6 \mathrm{mg}$ PDL, febrile neutropenia was less than G-CSF (13\% versus 20\%) [3]. Every chemotherapeutic regimen can cause neutropenia, but when the absolute neutrophil counts down below 1000, it causes the risk of fever neutropenia and sepsis which is very high. So most of the authors believe that GCF should be used to prevent the decrease of absolute neutrophil count below 1000 .

In a study that examined women who received chemotherapy during pregnancy and received G-CSF and PDL, there was no significant change in the age of birth, embryonic anomalies or child weight and in these patients; myelopoiesis is stopped at the first stage of growth (the promyelocyte/myelocyte stage) [17]. In a study by Calderwood et al. in 2001, splenomegaly was reported in all patients and mild hyperplastic hypertrophy was observed in a few others, and no short-term drug toxicity was observed [2]. As in the previous study stated, these generic drugs which were used in this study have been compared to brand type in previous studies. The authors stated the ANC and platelet count charts have the most valuable things in our curves. But after these, the lymphocyte count is valuable too. The authors chose headache, bone pain and the injection site reaction as the most common side effects of these drugs. we also mentioned other side effects but these three are the most important types which are needed to scrutinize.

\section{Conclusions}

Regarding our results, PDL is completely non-inferior in efficacy and also less toxic compared to PDG. Prescribing in single-dose and lower expenses of PDL introduces it as a cost-effective drug in the treatment of chemotherapy-induced neutropenia.

\section{Declarations}




\section{Funding}

A grant from Pooyesh Darou Company funded the leading research.

\section{Conflicts of interest/Competing interests}

Authors have no conflict of interest.

\section{Ethics approval}

This research was approved and registered in the Ethics Committee of Breast Cancer Research Center with code number: IR.ACECR.IBCRC.REC.1395.19. Also,

\section{Consent to participate}

All participants signed a written Informed Consent.

\section{Consent for publication}

Not applicable

\section{Availability of data and materials}

All data and materials are available for the journal.

\section{Code availability}

This study was registered in the Iranian Registry of Clinical Trials (IRCT) in https://www.irct.ir/ with registration code: IRCT20190504043465N1

\section{Authors' contributions}

SN and SH contributed to designing and supervising the project, data analysis, interpretation of data. MA and VK contributed to data collection, managing the project. All authors have participated in providing the first draft of the manuscript and they have approved the final version.

\section{Competing interests}

The authors declare that they have no conflict of interest.

\section{Acknowledgments}


The researchers at the Breast Cancer Research Center appreciate the financial support of Pooyesh Darou Company in providing the drug for two-arm of study.

\section{References}

1. Touw IP, Bontenbal M (2007) Granulocyte colony-stimulating factor: key (f) actor or innocent bystander in the development of secondary myeloid malignancy? Oxford University Press,

2. Calderwood S, Kilpatrick L, Douglas SD, Freedman M, Smith-Whitley K, Rolland M, Kurtzberg J (2001) Recombinant human granulocyte colony-stimulating factor therapy for patients with neutropenia and/or neutrophil dysfunction secondary to glycogen storage disease type 1b. Blood, The Journal of the American Society of Hematology 97 (2):376-382

3. Green M, Koelbl H, Baselga J, Galid A, Guillem V, Gascon P, Siena S, Lalisang R, Samonigg H, Clemens M (2003) A randomized double-blind multicenter phase III study of fixed-dose single-administration pegfilgrastim versus daily filgrastim in patients receiving myelosuppressive chemotherapy. Annals of Oncology 14 (1):29-35

4. Zeidler C, Schwinzer B, Welte K (2000) Severe congenital neutropenia: trends in diagnosis and therapy. Klinische Padiatrie 212 (4):145

5. Freedman MH (1997) Safety of long-term administration of granulocyte colony-stimulating factor for severe chronic neutropenia. Current opinion in hematology 4 (3):217-224

6. Bernini JC, Wooley R, Buchanan GR (1996) Low-dose recombinant human granulocyte colony-stimulating factor therapy in children with symptomatic chronic idiopathic neutropenia. The Journal of pediatrics 129 (4):551-558

7. Bonilla MA, Dale D, Zeidler C, Last L, Reiter A, Ruggeiro M, Davis M, Koci B, Hammond W, Gillio A (1994) Long-term safety of treatment with recombinant human granulocyte colony-stimulating factor (r-metHuG-CSF) in patients with severe congenital neutropenias. British journal of haematology 88 (4):723-730

8. Dale DC (1995) Hematopoietic growth factors for the treatment of severe chronic neutropenia. Stem cells 13 (2):94-100

9. Donadieu J, Leblanc T, Meunier BB, Barkaoui M, Fenneteau O, Bertrand Y, Maier-Redelsperger M, Micheau M, Stephan JL, Phillipe N (2005) Analysis of risk factors for myelodysplasias, leukemias and death from infection among patients with congenital neutropenia. Experience of the French Severe Chronic Neutropenia Study Group. haematologica $90(1): 45-53$

10. Leung T, Li C, Kwok K, Chik K, Shing M, Yuen P (2001) Severe chronic neutropenia in Chinese children in Hong Kong. Journal of paediatrics and child health 37 (2):137-141

11. Mempel K, Pietsch T, Menzel T, Zeidler C, Welte K (1991) Increased serum levels of granulocyte colony-stimulating factor in patients with severe congenital neutropenia.

12. Welte K, Dale D (1996) Pathophysiology and treatment of severe chronic neutropenia. Annals of hematology 72 (4):158-165

13. Donini M, Fontana S, Savoldi G, Vermi W, Tassone L, Gentili F, Zenaro E, Ferrari D, Notarangelo LD, Porta F (2007) G-CSF treatment of severe congenital neutropenia reverses neutropenia but does not correct the underlying functional deficiency of the neutrophil in defending against microorganisms. Blood 109 (11):4716-4723

14. Cottle TE, Fier CJ, Donadieu J, Kinsey SE Risk and benefit of treatment of severe chronic neutropenia with granulocyte colony-stimulating factor. In: Seminars in hematology, 2002. vol 2. Elsevier, pp 134-140

15. Ruef C, Coleman D (1991) GM-CSF and G-CSF: cytokines in clinical application. Schweizerische medizinische Wochenschrift 121 (12):397

Page $17 / 20$ 
16. Naparstek E (1995) Granulocyte colony-stimulating factor, congenital neutropenia, and acute myeloid leukemia. Mass Medical Soc,

17. Cardonick E, Irfan F, Torres N (2012) The use of Neupogen (filgrastim) or Neulasta (pegfilgrastim) during pregnancy when chemotherapy is indicated for maternal cancer treatment.

18. Dale DC, Bonilla MA, Davis MW, Nakanishi AM, Hammond WP, Kurtzberg J, Wang W, Jakubowski A, Winton E, Lalezari P (1993) A randomized controlled phase III trial of recombinant human granulocyte colony-stimulating factor (filgrastim) for treatment of severe chronic neutropenia.

19. Lehrnbecher $\mathrm{T}$ (2001) Hematopoietic growth factors in prophylaxis and therapy of infections complications in children with neutropenia. Klinische Padiatrie 213 (4):212

20. Sotomatsu M, Kanazawa T, Ogawa C, Watanabe T, Morikawa A (2000) Complication of rapidly progressive glomerulonephritis in severe congenital neutropenia treated with long-term granulocyte colony-stimulating factor (filgrastim). British journal of haematology 110 (1):234-235

21. Niemeyer C, Kratz C (2005) Is granulocyte colony-stimulating factor therapy a risk factor for myelodysplasia/leukemia in patients with congenital neutropenia? Haematologica 90 (1):2-3

22. Zeidler C, Welte K, Barak Y, Barriga F, Bolyard A, Boxer L, Cornu G, Cowan M, Dale D, Flood T (2000) Stem cell transplantation in patients with severe congenital neutropenia without evidence of leukemic transformation. Blood, The Journal of the American Society of Hematology 95 (4):1195-1198

23. Badolato R, Fontana S, Notarangelo LD, Savoldi G (2004) Congenital neutropenia: advances in diagnosis and treatment. Current opinion in allergy and clinical immunology 4 (6):513-521

24. Holmes F, Jones S, O’shaughnessy J, Vukelja S, George T, Savin M, Richards D, Glaspy J, Meza L, Cohen G (2002) Comparable efficacy and safety profiles of once-per-cycle pegfilgrastim and daily injection filgrastim in chemotherapy-induced neutropenia: a multicenter dose-finding study in women with breast cancer. Annals of Oncology 13 (6):903-909

25. Holmes F, O’shaughnessy J, Vukelja S, Jones S, Shogan J, Savin M, Glaspy J, Moore M, Meza L, Wiznitzer I (2002) Blinded, randomized, multicenter study to evaluate single administration pegfilgrastim once per cycle versus daily filgrastim as an adjunct to chemotherapy in patients with high-risk stage II or stage III/IV breast cancer. Journal of Clinical Oncology 20 (3):727-731

26. Rajan RS, Li T, Aras M, Sloey C, Sutherland W, Arai H, Briddell R, Kinstler O, Lueras AM, Zhang Y (2006) Modulation of protein aggregation by polyethylene glycol conjugation: GCSF as a case study. Protein science 15 (5):10631075

27. Wang Y-J, Hao S-J, Liu Y-D, Hu T, Zhang G-F, Zhang X, Qi Q-S, Ma G-H, Su Z-G (2010) PEGylation markedly enhances the in vivo potency of recombinant human non-glycosylated erythropoietin: a comparison with glycosylated erythropoietin. Journal of controlled release 145 (3):306-313

28. Li X-Q, Lei J-D, Su Z-G, Ma G-H (2007) Comparison of bioactivities of monopegylated rhG-CSF with branched and linear mPEG. Process Biochemistry 42 (12):1625-1631

29. da Silva Freitas D, Spencer PJ, Vassão RC, Abrahão-Neto J (2010) Biochemical and biopharmaceutical properties of PEGylated uricase. International journal of pharmaceutics 387 (1-2):215-222

30. Bailon P, Berthold W (1998) Polyethylene glycol-conjugated pharmaceutical proteins. Pharmaceutical Science \& Technology Today 1 (8):352-356

31. Tiwari K, Kattavarapu K, Shebannavar SN, Pokalwar S, Mishra MK, Chauhan UKS (2011) Evaluation of pegylation reaction and purification of monopegylated recombinant human granulocyte colony stimulating factor. 
32. Choi LMR, Guelcher C, Guerrera MF (2007) Novel treatment for severe congenital neutropenia with pegfilgrastim. Blood, The Journal of the American Society of Hematology 110 (12):4134-4134

33. Lähteenmäki PM, Jahnukainen K, Pelliniemi TT, Kainulainen L, Salmi TT (2009) Severe congenital neutropenia and pegfilgrastim. European journal of haematology 82 (1):75-76

34. Bruns I, Steidl U, Fischer JC, Czibere A, Kobbe G, Raschke S, Singh R, Fenk R, Rosskopf M, Pechtel S (2008) Pegylated granulocyte colony-stimulating factor mobilizes CD34+ cells with different stem and progenitor subsets and distinct functional properties in comparison with unconjugated granulocyte colony-stimulating factor. haematologica 93 (3):347-355

\section{Figures}

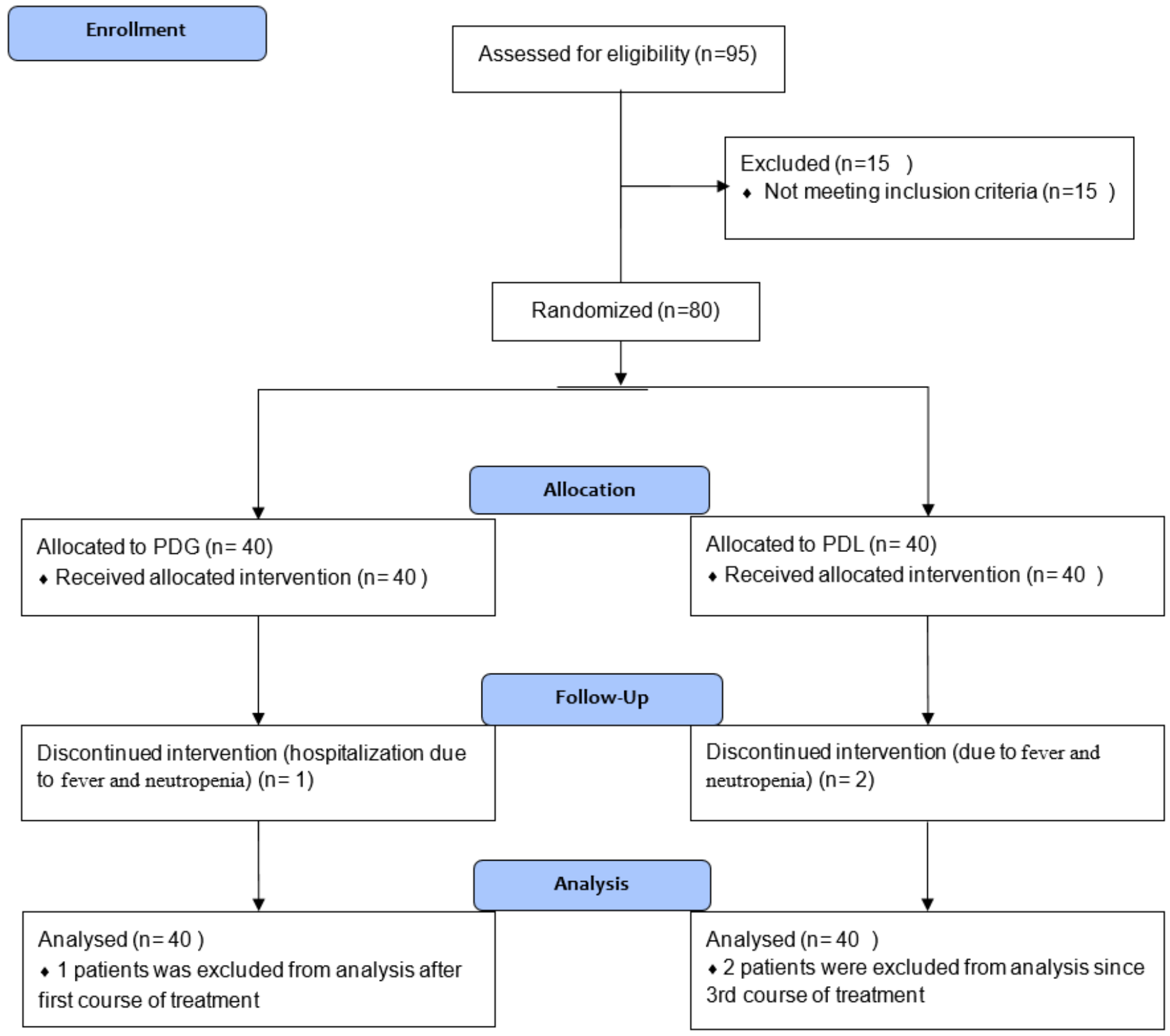

Figure 1 


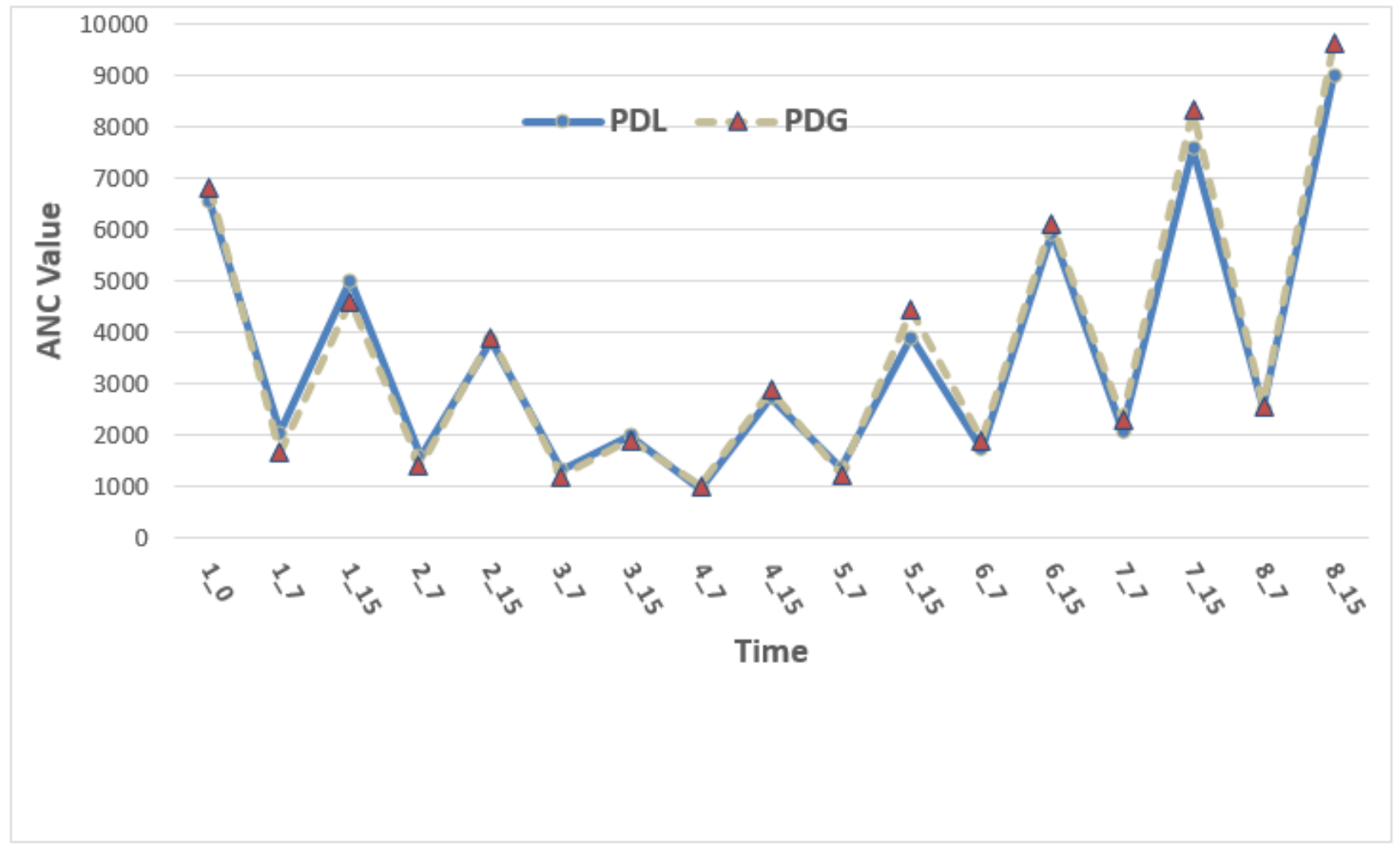

Figure 2

Changes of ANC values during eight courses of chemotherapy between two groups

\section{Supplementary Files}

This is a list of supplementary files associated with this preprint. Click to download.

- CONSORT2010Checklist.doc 